\title{
Beclin1-armed oncolytic Vaccinia virus enhances the therapeutic efficacy of R-CHOP against lymphoma in vitro and in vivo
}

\author{
SHUFANG XIE ${ }^{1,2^{*}}$, WEIMIN FAN ${ }^{2,3^{*}}$, CHEN YANG $^{2,4}$, WEN LEI $^{5}$, HONGYING PAN $^{6}$, \\ XIANGMIN TONG ${ }^{2,7}$, YI WU ${ }^{8}$ and SHIBING WANG ${ }^{2,7}$ \\ ${ }^{1}$ The Second Clinical Medical College, Zhejiang Chinese Medical University, Hangzhou 310000; \\ ${ }^{2}$ Molecular Diagnosis Laboratory, Zhejiang Provincial People's Hospital, People's Hospital of Hangzhou Medical College, \\ Hangzhou, Zhejiang 310014; ${ }^{3}$ Department of Center for Reproductive Medicine, Taihe Hospital, \\ Affiliated to Hubei University of Medicine, Shiyan, Hubei 442000; ${ }^{4}$ Department of Clinical Medicine, Qingdao University, \\ Qingdao, Shandong 266071; ${ }^{5}$ Department of Hematology, The Second Affiliated Hospital, College of Medicine, \\ Zhejiang University, Hangzhou 310003; ${ }^{6}$ Department of Infectious Diseases, Zhejiang Provincial People's Hospital, \\ People's Hospital of Hangzhou Medical College, Hangzhou, Zhejiang 310014; \\ ${ }^{7}$ The Key Laboratory of Tumor Molecular Diagnosis and Individualized Medicine of Zhejiang Province, \\ Zhejiang Provincial People's Hospital; ${ }^{8}$ Department of Hematology, Zhejiang Provincial People's Hospital, \\ People's Hospital of Hangzhou Medical College, Hangzhou, Zhejiang 310014, P.R. China
}

Received June 11, 2020; Accepted December 4, 2020

DOI: $10.3892 /$ or.2021.7942

\begin{abstract}
Non-Hodgkin lymphoma (NHL) is a form of lymphoid malignancy, with diffuse large B cell lymphoma (DLBCL) being the most common NHL isoform. Approximately half of patients with DLBCL are successfully cured via first-line Rituximab, Cyclophosphamide, Epirubicin, Vindesine, Prednisolone (R-CHOP) treatment. However, $30-40 \%$ of patients with DLBCL ultimately suffer from treatment-refractory or relapsed disease. These patients often suffer from high mortality rates owing to a lack of suitable therapeutic options, and all patients are at a high risk of serious treatment-associated dose-dependent toxicity. As such,
\end{abstract}

Correspondence to: $\mathrm{Dr} \mathrm{Yi} \mathrm{Wu}$, Department of Hematology, Zhejiang Provincial People's Hospital, People's Hospital of Hangzhou Medical College, 158 Shangtang Road, Hangzhou, Zhejiang 310014, P.R. China

E-mail: wuyi10000@126.com

Dr Shibing Wang, Molecular Diagnosis Laboratory, Zhejiang Provincial People's Hospital, People's Hospital of Hangzhou Medical College, 158 Shangtang Road, Hangzhou, Zhejiang 310014, P.R. China

E-mail: wangshibing@hmc.edu.cn

*Contributed equally

Abbreviations: NHL, non-Hodgkin lymphoma; OVV, oncolytic Vaccinia virus; DLBCL, diffuse large B cell lymphoma; R-CHOP, Rituximab, Cyclophosphamide, Epirubicin, Vindesine, Prednisolone

Key words: OVV, Beclin1, gene therapy, autophagic cell death, R-CHOP, lymphoma it is essential to develop novel treatments for NHL that are less toxic and more efficacious. Oncolytic Vaccinia virus (OVV) has shown promise as a means of treating numerous types of cancer. Gene therapy strategies further enhance OVV-based therapy by improving tumor cell recognition and immune evasion. Beclin1 is an autophagy-associated gene that, when upregulated, induces excess autophagy and cell death. The present study aimed to develop an OVV-Beclin1 therapy capable of inducing autophagic tumor cell death. OVV-Beclin1 was able to efficiently kill NHL cells and to increase the sensitivity of these cells to R-CHOP, thereby decreasing the dose-dependent toxic side effects associated with this chemotherapeutic regimen. The combination of OVV-Beclin1 and R-CHOP also significantly improved tumor growth inhibition and survival in a BALB/c murine model system owing to the synergistic induction of autophagic cell death. Together, these findings suggest that OVV-Beclin1 infection can induce significant autophagic cell death in NHL, highlighting this as a novel means of inducing tumor cell death via a mechanism that is distinct from apoptosis and necrosis.

\section{Introduction}

Non-Hodgkin lymphoma (NHL) is a form of lymphoid malignancy that can manifest in lymph nodes and other lymphatic organs (1). NHL is one of the 10 most common forms of cancer globally, with $\sim 500,000$ newly diagnosed cases annually. There are $\geq 40$ major subtypes of NHL with various genetic, clinical, and morphological features. Diffuse large B cell lymphoma (DLBCL) is the most common form of NHL, accounting for $30-40 \%$ of new cases. Rituximab, Cyclophosphamide, Epirubicin, Vindesine, Prednisolone (R-CHOP) is the first-line standard of care treatment for these patients and can achieve a $60 \%$ overall survival (OS) rate (2); however, up to $40 \%$ of 
patients suffer from treatment-refractory or relapsed disease with associated high mortality rate $(3,4)$.Chemotherapeutic drugs can cause extensive DNA damage within both tumor and healthy cells (5), such that even patients that survive chemotherapy suffer significant systemic toxicity and renal failure in a dose-dependent manner (6).

A number of alternative therapeutic regimens have been developed to overcome the aforementioned limitations and toxicity of standard R-CHOP therapy, including oncolytic Vaccinia virus (OVV) and gene therapies (7-9). OVV approaches allow for direct tumor cell targeting, whereas gene therapy allows precise genetic manipulation in target cells. A previous OVV and gene therapy-based study have focused on the induction of apoptosis within tumor cells (10). However, apoptosis is a complex process and certain tumor cells are resistant to apoptotic cell death (11), limiting the potential utility of correlative therapeutic strategies. In addition to apoptotic death, cells can also undergo autophagic death. Under normal physiological conditions, autophagy is a process whereby cells respond to stress and maintain homeostasis and proper protein folding by degrading intracellular protein aggregates and organelles $(12,13)$. Beclin1 is a key regulator of autophagy that facilitates co-localization of other autophagic proteins to promote autophagosome formation and maturation in mammalian cells (14). Beclin1 has been found to function as a tumor suppressor gene in breast, ovarian and other types of cancer $(15,16)$. The specific degree of autophagy is important: Moderate levels of autophagy improve cell survival under stressful conditions, whereas excessive autophagy triggers cell death (17-19). Chemotherapeutic drugs cause extensive DNA damage, but tumor cells can develop resistance to such damage by modulating intracellular DNA repair capability (20). Increasing autophagic activity within tumor cells can lead to the degradation of these organelles and proteins associated with DNA repair, potentially increasing their susceptibility to chemotherapy (21-23)

The present study investigated a potential therapeutic strategy aimed at increasing intratumoral Beclin1 expression levels as a means of inducing autophagic cell death, based on previous research (24-26). OVV-Beclin1 was developed as a therapeutic approach to induce NHL cell death and increasing sensitivity to chemotherapeutic treatment. Levels of the autophagosome marker LC3B in NHL cells were assessed via western blotting following OVV-Beclin1 treatment to confirm efficacy. Tumor inhibitory effects of OVV-Beclin1 treatment were confirmed via MTT and colony formation assays, as well as an in vivo murine model system.

\section{Materials and methods}

Human samples, cell lines and reagents. Pathological biopsy and paracancerous tissue samples were obtained from 10 patients with NHL that underwent fine-needle aspiration and biopsy at Zhejiang Provincial People's Hospital (Hangzhou, China) from January 2015 to December 2016. All samples were collected in a manner consistent with Chinese law and the study was approved by the Ethics Committee of Zhejiang People's Hospital (approval no. 2019KY232), and all patients provided written informed consent. As DLBCL tumors are invasive, paracancerous tissue samples were selected from the same region as the primary tumor (distance, $>5 \mathrm{~cm}$ ).

OCI-LY3 and Pfeiffer cells were obtained from the Cell Bank of the Chinese Academy of Sciences (Shanghai, China), and were grown in RPMI-1640 (HyClone; GE Healthcare Life Sciences) containing 10\% FBS (Gibco; Thermo Fisher Scientific, Inc.) and $1 \%$ penicillin/streptomycin. Additionally, 293 cells were obtained from the Institute of Clinical Medicine of Zhejiang Provincial People's Hospital and maintained in DMEM containing 10\% FBS, 2 mM L-glutamine (both Gibco; Thermo Fisher Scientific, Inc.) and penicillin/streptomycin. Cells were cultured in a humidified $5 \% \mathrm{CO}_{2}$ incubator at $37^{\circ} \mathrm{C}$.

Anti-Beclin1 (1:1,000; clone 2A4; cat. no. 4122), anti-LC3A/B (1:1,000; clone D3U4C; cat. no. 13118) and anti-P62 (1:1,000; clone Ser403; cat. no. 39786) were obtained from Cell Signaling Technology, Inc., while anti-caspase-3 (1:500; cat. no. ab13847) was from Bioworld Technology. Anti-PARP (1:500; cat. no. 100984-T46) was purchased Sino Biological, Inc. and anti- $\beta$-actin (1:1,000; cat. no. ER62585) was from Huangzhou HuaAn Biotechnology Co., Ltd. Acridine orange stain was obtained from Vacutainer (Becton-Dickinson and Company). For R-CHOP, Rituximab was from Roche Diagnostics $\mathrm{GmbH}$, Cyclophosphamide was from Baxter Oncology $\mathrm{GmbH}$, Epirubicin was from Shenzhen Main Luck Pharmaceuticals, Inc., Vindesine was from Yangtze River Pharmaceutical Group Co., Ltd. and Prednisolone was from Pfizer, Inc.

Immunohistochemistry (IHC) and hematoxylin and eosin $(H \& E)$ staining. Samples from patients with DLCBL $(n=10$; median age: 70 ; range: $55-81$ years) were fixed with $10 \%$ formalin at room temperature for $>24 \mathrm{~h}$, embedded in paraffin and cut into $4-\mu \mathrm{m}$ sections. Sections were deparaffinized with xylene, rehydrated using an ethanol gradient $(100,95,90,80$ and $70 \%$ ) and antigen recovery was performed using sodium citrate buffer at $121^{\circ} \mathrm{C}$ and $80 \mathrm{kPa}$. Endogenous peroxidase activity was quenched using a $3 \% \mathrm{H}_{2} \mathrm{O}_{2}$ solution to treat samples for $15 \mathrm{~min}$ at room temperature. Following three washes in PBS, sections were stained with anti-Beclin1 (1:200) overnight at $4^{\circ} \mathrm{C}$. Sections were then washed and stained with appropriate horseradish peroxidase (HRP)-labeled secondary antibodies for $30 \mathrm{~min}$ at room temperature, as previously described (16). Sections were again washed with PBS, stained for $15 \mathrm{~min}$ with 3'3'-diaminobenzidine at room temperature, and then counterstained with hematoxylin at room temperature for $4 \mathrm{~min}$. Sections were examined via light microscopy (Shanghai Laika Microscope Co., Ltd.; magnification, x400) and were then assessed with Image-Pro Plus software (Image-Pro Express 6.0; Media Cybernetics, Inc.). A total of five fields of view per sample was analyzed. After fixing the tissue as aforementioned, the tissue was dyed with hematoxylin for $10 \mathrm{~min}$, rinsed with PBS three times ( 3 min each), washed with running water, dyed with eosin for $2 \mathrm{~min}$, rinsed with PBS three times (3 min each), dehydrated with alcohol and xylene and finally sealed (all at room temperature), observed and photographed under a light microscope.

OVV-Beclinl preparation and characterization. A homologous recombination approach was used for OVV and OVV-Beclin1 preparation, as previously described (27-29). The 
complete Beclin1 gene sequence was amplified via PCR from the plasmid (cat. no. HG11162-ACG; Sino Biological, Inc.) with the following primers: Forward, 5'-CCGGAATTCACCATG GAAGGGTCTAAGACGTCCAAC-3' and reverse, 5'-ACG CGTCGACTTATCATTTGTTATAAAATTGTGAGG-3'. The PCR conditions were as follows: Initial denaturation, $95^{\circ} \mathrm{C}$, $3 \mathrm{~min}$; denaturation, $95^{\circ} \mathrm{C}, 45 \mathrm{sec}$; annealing, $55^{\circ} \mathrm{C}, 45 \mathrm{sec}$; elongation, $72^{\circ} \mathrm{C}, 90 \mathrm{sec}\left(30\right.$ cycles) and final extension, $72^{\circ} \mathrm{C}$, $5 \mathrm{~min}$. This sequence was then inserted into the pCB plasmid via molecular cloning. The sequence was verified, after which pCB or pCB-Beclin1 underwent homologous recombination with wild-type Vaccinia virus in 293A cells, as previously described (28). When significant infection and associated suppression effects were evident, as previously described (30), these 293 cell cultures were expanded and used to harvest prepared virus particles. Viral particles were then purified by repeatedly freezing and thawing (freezing temperature, $-80^{\circ} \mathrm{C}$; thawing temperature, $37^{\circ} \mathrm{C} ; 3$ cycles at $15 \mathrm{~min}$ each). Cell samples followed by ultracentrifugation $\left(750 \mathrm{x} \mathrm{g} ; 4^{\circ} \mathrm{C} ; 10 \mathrm{~min}\right)$. The median tissue culture infective dose for these OVV particles was then measured.

Acridine orange staining. Acridine orange was used to stain acidic autophagosomes within NHL cells, as previously described $(31,32)$. Briefly, these cells were treated with PBS, OVV, or OVV-Beclin1 (MOI, 50) for $48 \mathrm{~h}$ at $37^{\circ} \mathrm{C}$ and were then stained with acridine orange for $15 \mathrm{~min}$. Cell fluoresce was then assessed via fluorescence microscopy (magnification, $\mathrm{x} 400$; excitation, $560 \mathrm{~nm}$; emission, $645 \mathrm{~nm}$ ) as previously described) (32).

Western blotting. Proteins in OCI-LY3 cells and murine tumor tissue were extracted using RIPA (Beyotime Institute of Biotechnology) buffer containing protease inhibitors, after which a BCA assay kit was used to measure protein levels in these samples. Equal amounts of protein $(10 \mu \mathrm{g})$ were then separated via SDS-PAGE (10-15\%), and samples were transferred to PVDF membranes and probed at $4^{\circ} \mathrm{C}$ overnight with appropriate primary antibodies. Membranes were blocked with $\sim 5 \%$ milk in TBST $(0.05 \%$ Tween-20) for $1 \mathrm{~h}$ at room temperature. Blots were then probed at room temperature for $1 \mathrm{~h}$ with secondary HRP-conjugated antibody (1:4,000; HuaAn Biotechnology Co., Ltd) for $1 \mathrm{~h}$. Protein bands were detected via enhanced chemiluminescence detection system (Thermo Fisher Scientific, Inc.) and quantification was performed using ImageJ software (1.48v; National Institutes of Health).

MTT assay. OCI-LY3 cells were seeded in 96-well plates ( $2 \times 10^{4}$ cells per well) and cultured at $37^{\circ} \mathrm{C}$ for $12 \mathrm{~h}$. PBS was used as the control. In order to assess cell death, cells were treated with the apoptosis inhibitor z-VAD and/or the autophagy inhibitor bafilomycin A1 (BAF1; both $10 \mu \mathrm{M}$; both Selleck Chemicals). Following incubation at $37^{\circ} \mathrm{C}$ for $72 \mathrm{~h}, 20 \mu \mathrm{l} \mathrm{MTT}$ reagent (Promega Corporation) was added to each well and cultured for $4 \mathrm{~h}$ at $37^{\circ} \mathrm{C}$ with $5 \% \mathrm{CO}_{2}$. Finally, the absorbance was detected at a wavelength of $490 \mathrm{~nm}$ using a Microplate Reader Model $550 \mathrm{~nm}$. (Bio-Rad Laboratories, Inc.).

Colony formation assay and growth curves. OCI-LY3 cells were infected with OVV-green fluorescent protein (GFP) or
OVV-Beclin1(MOI=50 and 100) in a 6-well plate for $12 \mathrm{~h}$ at $37^{\circ} \mathrm{C}$, and the cell density is $10^{5} /$ well. After which they were washed twice in PBS, seeded in 24-well plates (density, $10^{4} / \mathrm{ml}$ ) in a solution containing $2.7 \%$ methylcellulose and $200 \mu \mathrm{l}$ FBS and cultured for 10 days at $37^{\circ} \mathrm{C}$, after which colonies (>50 cells) were counted via light microscopic examination. (magnification, $\mathrm{x} 10$ ).

Murine xenograft experiments. Female nude mice $(n=25$; age, 5 weeks; weight, $23.5 \mathrm{~g}$ ) were raised in the Experimental Animal Center of the Chinese Academy of Sciences (Shanghai, China) received a subcutaneous right flank injection of $1 \times 10^{7}$ OCI-LY3 cells. Mice received standard diet and water ad libitum and were housed under specific pathogen-free conditions. Beginning on day 9 after injection of OCY-LY3 cells, when tumors were $\sim 100 \mathrm{~mm}^{3}$, tumor volume was measured and animals were randomized into five treatment groups (n=5/group): PBS $(100 \mu \mathrm{l}), \mathrm{OVV}\left(10^{8}\right.$ PFU/animal; intratumoral injection), OVV-Beclin1 $\left(10^{8}\right.$ PFU/animal; intratumoral injection), R-CHOP (Rituximab, 375.0; Cyclophosphamide, 750.0; Epirubicin, 50.0; Vindesine, 1.4; Prednisolone, $15.0 \mathrm{mg} / \mathrm{m}^{2}$; caudal vein injection) and $\mathrm{R}-\mathrm{CHOP}+\mathrm{OVV}-\mathrm{Beclin} 1$ (administered with the same dosing strategies as in the R-CHOP-alone and OVV-Beclin1-alone groups). For mice administered R-CHOP therapy, Rituximab $(2.74 \mathrm{mg}$ ) was administered on day 1 (the day on which tumor volume reached $\sim 100 \mathrm{~mm}^{3}$ ) CHO $(5.40,0.36$ and $0.01 \mathrm{mg}$, respectively) was administered on day 2 (the day after R-CHOP therapy) and Prednisolone $(0.11 \mathrm{mg})$ administered on days 2-5. For animals treated with both OVV-Beclin1 and R-CHOP, OVV-Beclin1 was administered prior to R-CHOP as aforementioned. Following treatment, tumor volume was monitored every other day using calipers and calculated as follows: $0.5 \times$ length $\mathrm{x}$ width ${ }^{2}$. After 30 days, mice were sacrificed via cervical dislocation. Tumors were harvested, fixed with $10 \%$ formalin, paraffin-embedded and used for downstream analysis as aforementioned. The Animal Center of Zhejiang Chinese Medical University (Hangzhou, China) approved the present study, which was performed in accordance with appropriate guidelines and regulations (33).

Statistical analysis. SPSS 17.0 software (SPSS, Inc.) was used for statistical analysis. Data are presented as the mean \pm SD of three independent repeats. Continuous data were compared via unpaired two-tailed Student's t-tests or two-way ANOVA followed by Tukey's multiple comparisons post hoc test as appropriate. The Kaplan-Meier approach and log-rank test were used to compare survival outcomes and Prism (version. no. 8.0.2; GraphPad Software, Inc.) was used to construct figures. $\mathrm{P}<0.05$ was considered to indicate a statistically significant difference.

\section{Results}

Clinical characteristics of patients with NHL. The clinical characteristics of patients with NHL are shown in Table I. Beclin1 expression levels in these NHL tumor tissue samples were assessed via IHC, revealing notably decreased Beclin1 levels in tumor cells compared with normal control cells (Fig. 1). Beclin1 staining (brown) was very weak in NHL 
Table I. Clinical characteristics of patients with non-Hodgkin lymphoma. Beclin1 levels were analyzed in 10 randomly selected patients with DLBCL, revealing no association between Beclin1 levels and patient sex, age or immunophenotype.

\begin{tabular}{clll}
\hline Patient & Sex/age, years & Classification & \multicolumn{1}{c}{ Immunohistochemical staining results } \\
\hline 1 & Female/81 & GCB & CD3(-), CD20(+), CD45(-), CD10(+), PAX5(+) \\
2 & Male/55 & Non-GCB & PAX5(++), CD45(-), CD10(-), BCL-6(+), MUMI(+) \\
3 & Male/62 & Non-GCB & CD3(-), CD20(+), CD45(-), CD10(-), BCL-6(-), MUMI(-) \\
4 & Male/64 & GCB & CD3(-), CD20(+), CD45(-), CD10(-), BCL-6(+), MUMI(-) \\
5 & Male/77 & Non-GCB & CD3(-), CD20(+), CD45(-), CD10(-), BCL-6(+), MUMI(+) \\
6 & Female/67 & Non-GCB & CD3(-), CD20(+), CD45(-), CD10(-), BCL-6(+), MUMI(+) \\
7 & Female/76 & Non-GCB & CD3(-), CD20(+), CD45(-), CD10(-), BCL-6(+), MUMI(+) \\
8 & Male/55 & Non-GCB & CD3(-), CD20(+), CD45(-), CD10(-), BCL-6(-), MUMI(+) \\
9 & Female/51 & Non-GCB & CD3(-), CD20(+), CD45(-), CD10(-), BCL-6(-), MUMI(+) \\
10 & Female/63 & Non-GCB & CD3(-), CD20(+), CD45(-), CD10(-), BCL-6(+), MUMI(+)
\end{tabular}

Pan B cell type: CD3(-), CD20(+), CD45(-), PAX5(+). GCB: CD10(+) or BCL-6(+), MUMI(-). Non-GCB: CD10(-), MUMI(+) or BCL-6(-), MUMI(-). DLBCL, diffuse large B cell lymphoma; GCB, germinal center type DLBCL.
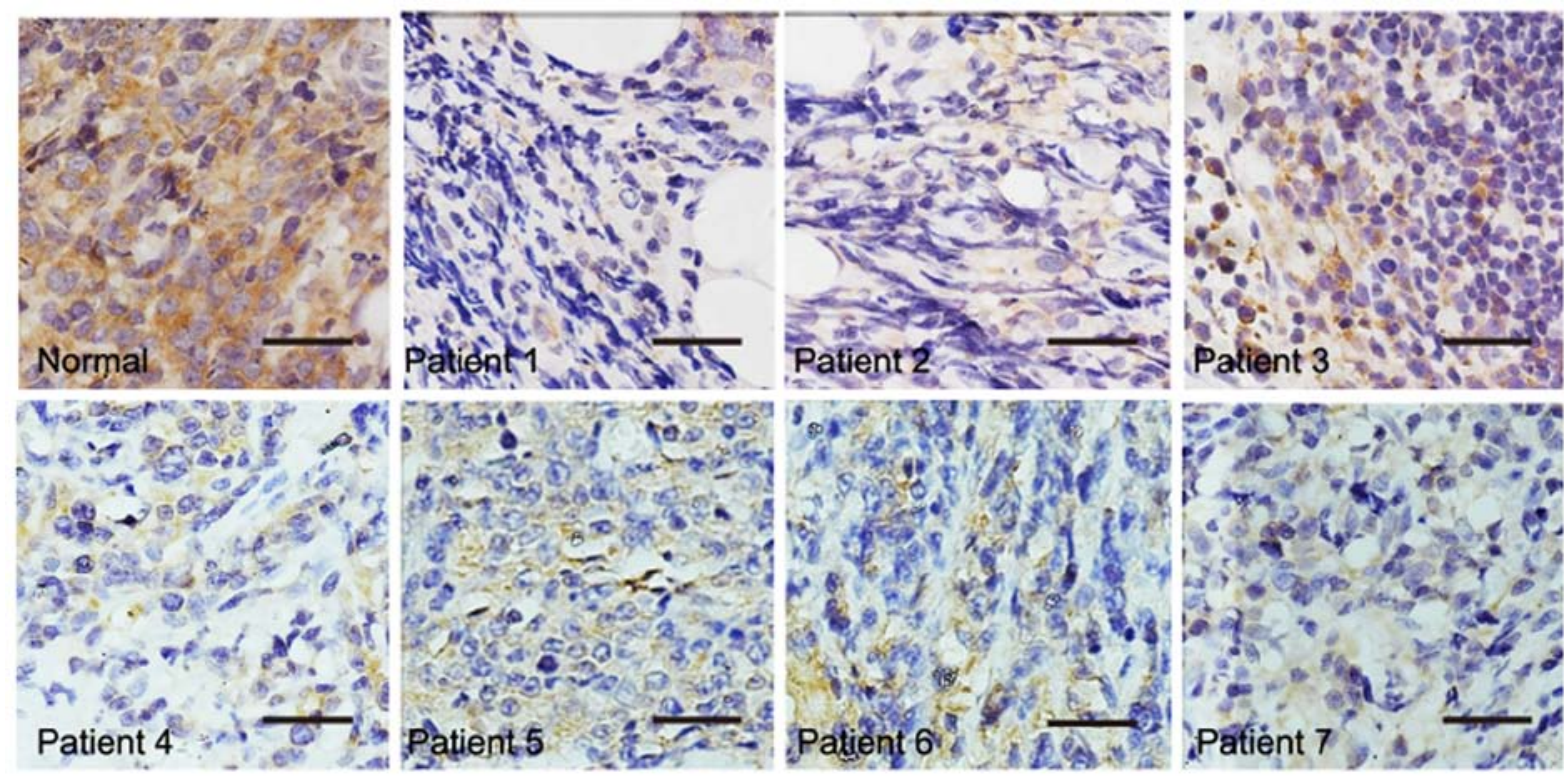

Figure 1. Beclin1 expression levels are decreased in patients with DLBCL. DLBCL (affected lymph nodes) and adjacent normal tissue were stained for Beclin1. Cytoplasmic Beclin1 levels (brown) were lower in DLBCL compared with paired normal tissue. Representative images are shown. Scale bar, $50 \mu \mathrm{m}$. DLBCL, diffuse large B cell lymphoma.

tissue, suggesting that Beclin1 downregulation may be associated with NHL progression.

Assessment of OVV-Beclin1 infectivity in NHL cells. OVV-Beclin1 was generated via homologous recombination (Fig. 2A). OVV infectivity was assessed using an OVV-GFP construct, which was used to infect the OCI-LY3 and Pfeiffer NHL cell lines. These cells were readily infected by this virus, with a dose-dependent increase in the frequency of GFP-positive cells from 30 to $60 \%$ on day 2 post-infection as viral MOI rose from 10 to 50 (Fig. 2B and C). Autophagic changes in cells were assessed. Western blotting demonstrated that the OVV-Beclin1 treatment was associated with notably increased Beclin1 and LC3B levels and decreased those of P62 (Fig. 2D), suggesting that cytotoxicity was associated with increased induction of autophagy. In order to confirm that the OVV-Beclin1 virus was capable of inducing autophagy in NHL cells, Beclin1 levels in these cells were measured via western blotting; OVV-Beclin1 led to a dose-dependent increase in levels of this protein in NHL cell preparations (MOI=10-50). By contrast, mock-infected cells exhibited relatively low levels of Beclin1 protein. Other autophagy-associated protein levels in these cells were assessed, including levels of LC3B, which forms during autophagy and is associated with the membrane of autophagosomes, and SQSTM1(P62), which is a ubiquitin-binding protein that exhibits decreased expression as autophagy progresses (34). Measurements of these proteins in OVV-Beclin1-infected NHL cells (Fig. 2E) further confirmed that the virus was able to effectively induce autophagy within NHL cells following infection. This indicates that these cells 
A

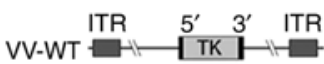

ITR 5'TK Pse/L P7.5 3'TK ITR

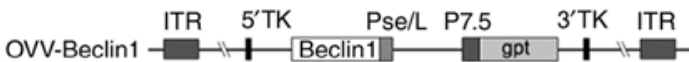

B

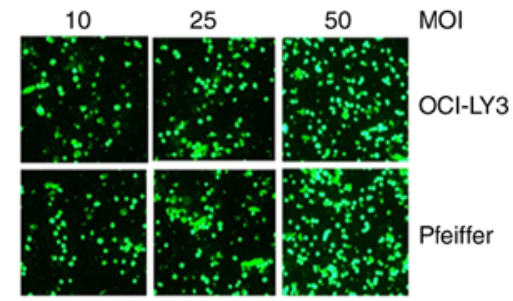

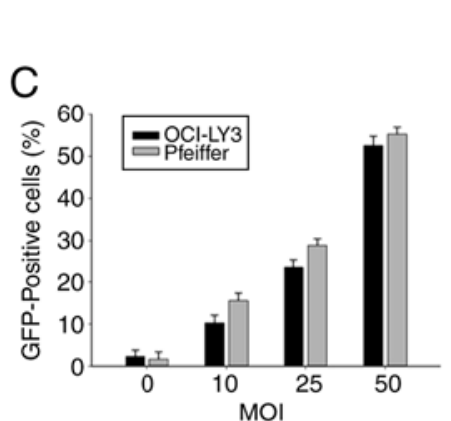

$\mathrm{F}$

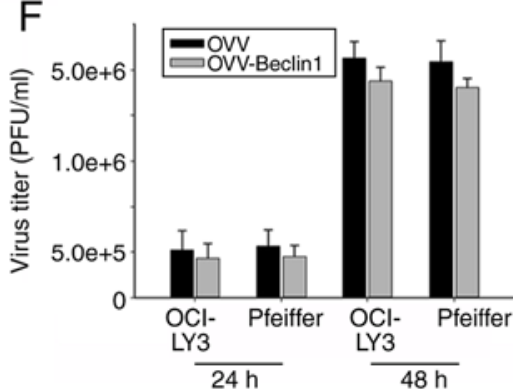

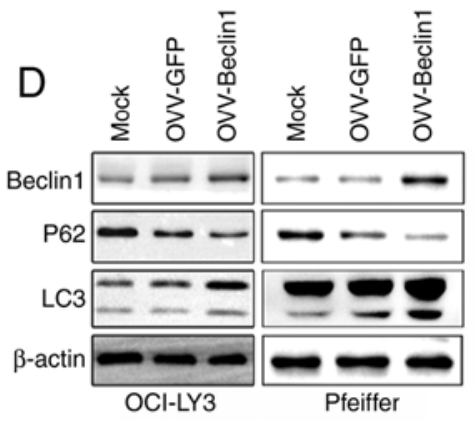

$\mathrm{E}$
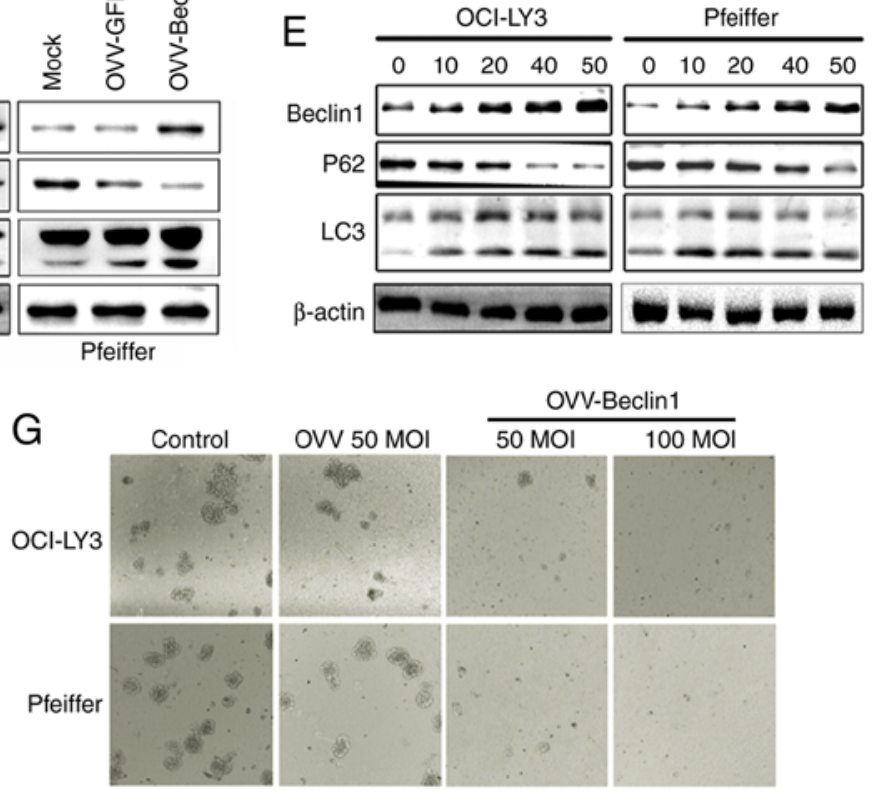

Figure 2. OVV-Beclin1 infects NHL cells. (A) Structure of OVV-Beclin1. OVV and OVV-Beclin1 were prepared via homologous recombination using VV-WT; Beclin1 expression cassette was introduced into the TK region of the virus. (B) OCI-LY3 and Pfeiffer cells were infected with OVV-GFP (MOI=10, 25 or 50) and analyzed via fluorescence microscopy after $24 \mathrm{~h}$ (magnification, x200). (C) Quantitative analysis of fluorescence microscopy results. (D) OCI-LY3 cells were treated with OVV or OVV-Beclin1 (MOI=50); after $48 \mathrm{~h}$, expression levels of Beclin1, LC3 and actin were measured via western blotting. (E) Western blotting was used to quantify levels of Beclin-1, LC3A/B, and P62 in NHL cells at $48 \mathrm{~h}$ post-OVV-Beclin1 infection. Actin was used as a loading control. (F) Determination of virus titer to determine the time of optimal infection. Detection of virus titers after infection 24 and 48 h. (G) OVV-Beclin1 inhibits NHL cell proliferation. Compared with the OVV group, OVV-Beclin1 significantly inhibited frontal growth of NHL cells. The inhibitory effect was greater as MOI increased from 50 to 100. OVV, oncolytic Vaccinia virus; NHL, non-Hodgkin lymphoma; WT, wild-type; TK, thymidine kinase; Pse/L, synthetic early/late promoter; P7.5, VV early-late promoter; gpt, mycophenolic acid resistance gene; ITR, inverted terminal repeat; GFP, green fluorescent protein.

were highly sensitive to OVV-Beclin1 infection. Infection with a 50 or 100 MOI dose of OVV-Beclin1 notably inhibited cell viability, as demonstrated by colony formation assay. In order to ensure the best infection effect, virus titer was tested during the experiment. The results showed that after $48 \mathrm{~h}$ infection, the virus titer was significantly higher than at $24 \mathrm{~h}$ (Fig. 2F and G).

OVV-Beclinl induces autophagic cell death in NHL. Having demonstrated that the OVV-Beclin1 was capable of efficiently infecting NHL cells and inducing autophagy, it was next determined whether this virus drove autophagic cell death. Levels of apoptosis-associated proteins were measured via western blotting; expression levels were not significantly altered following OVV-Beclin1 infection (Fig. 3A). In further support of this result, treatment of NHL cells with the apoptosis inhibitor z-VAD did not prevent OVV-Beclin1-induced cell death (Fig. 3B), indicating that death occurred in a caspase-independent manner. During autophagy, acidic autophagosomes form and interact with microtubule-associated protein LC3B (35). Autophagic vesicles were therefore detected via staining with acid-sensitive acridine orange (Fig. 3C). These autophagosomes contained protein, debris and organelles such as mitochondria, confirming successful OVV-Beclin1-mediated autophagic induction. Treating these cells with the autophagy inhibitor BAF1 prevented OVV-Beclin1-induced cell death. Together, these findings confirmed the ability of OVV-Beclin1 to induce autophagy in NHL cells, with BAF1 inhibiting this process (Fig. 3D).

OVV-Beclinl and R-CHOP exhibit synergistic efficacy in vivo. The aforementioned results were next assessed in vivo to determine whether OVV-Beclin1 in combination with R-CHOP may be a viable treatment for NHL. A murine NHL xenograft model was established by subcutaneously implanting BALB/c nude mice with OCI-LY3 cells. After tumors had grown to $\sim 100 \mathrm{~mm}^{3}$, animals were randomized into 5 treatment groups (PBS, OVV, OVV-Beclin1, R-CHOP and OVV-Beclin1 + R-CHOP). OVV-Beclin1 was administered intratumorally every third day over the course of three treatment cycles and PBS and R-CHOP were injected via the caudal vein. Tumor volume in these mice was then monitored $\geq 3$ times per week, and animals exhibiting signs of 
A
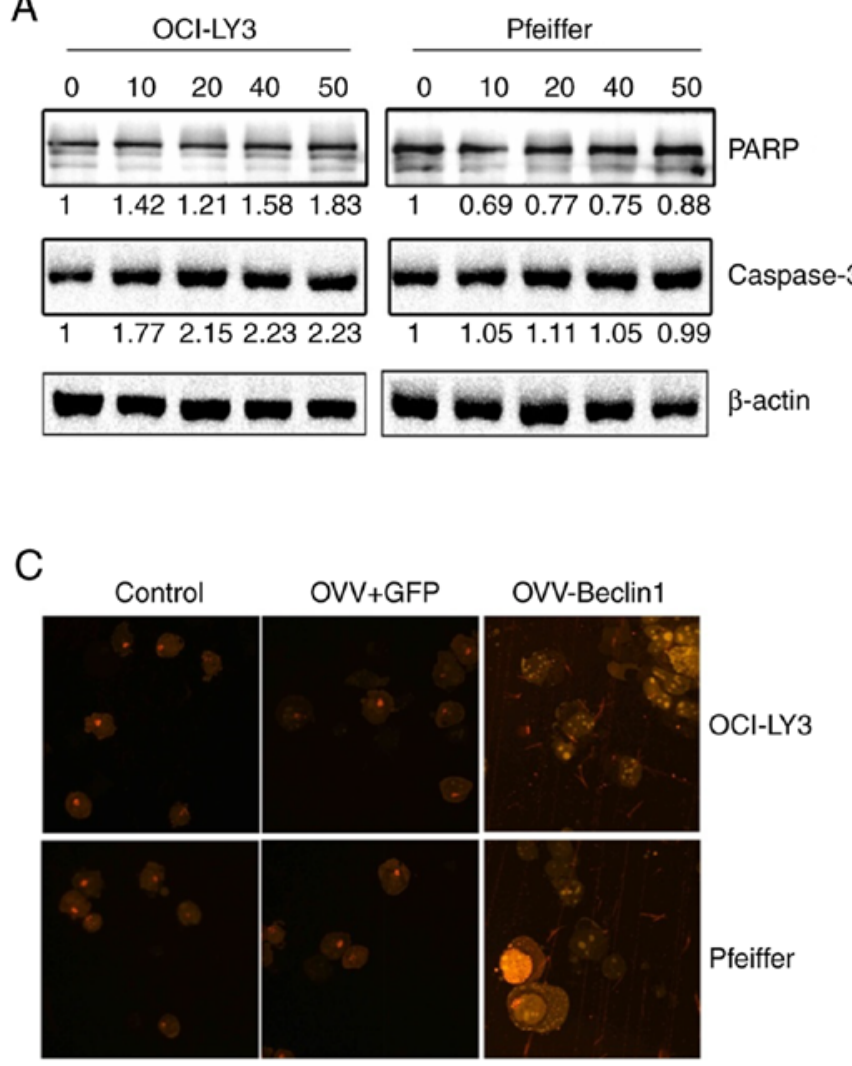

$\mathrm{B}$

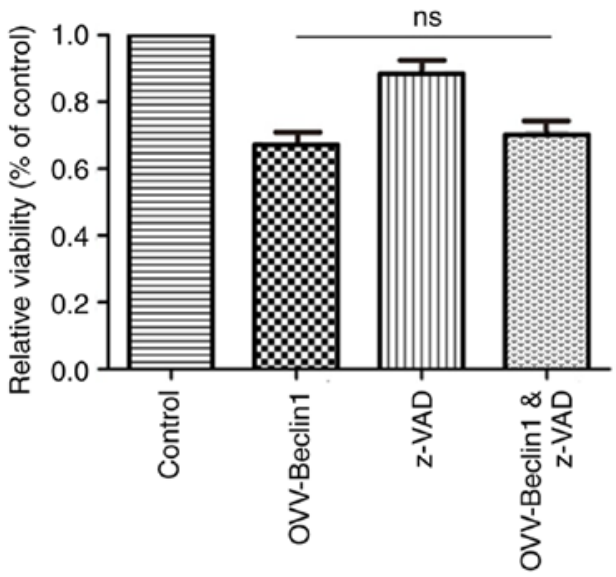

D

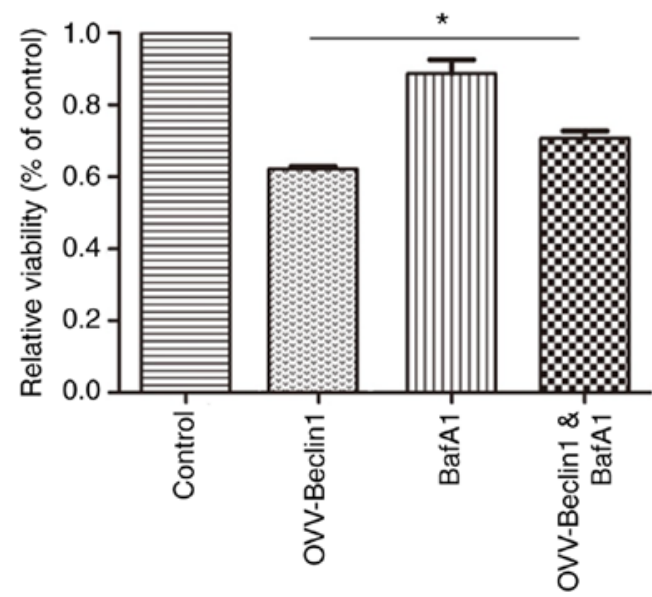

Figure 3. OVV-Beclin1 induces autophagic death of NHL cells. (A) Levels of apoptosis-associated proteins (PARP and caspase-3) were assessed via western blotting using OCI-LY3 and Pfeiffer cells infected with OVV-Beclin1 (MOI=0, 10, 25 or 50). Actin was used as a loading control. (B) OCI-LY3 cells were treated with OVV-Beclin1 in the presence or absence of z-VAD $(10 \mu \mathrm{M})$ for $72 \mathrm{~h}$, after which MTT assay was used to measure viability. (C) Autophagosomes in NHL cells were stained using acridine orange following treatment with PBS, OVV or OVV-Beclin1 (MOI=50) for 48 h. Finally, fluorescence was observed under a microscope (magnification, $\mathrm{x} 400$ ). (D) OCI-LY3 cells were infected with OVV-Beclin1 (MOI=50) in the presence or absence of $10 \mu \mathrm{M}$ BafA1 Viability was assessed via MTT assay. ${ }^{*} \mathrm{P}<0.05$. OVV, oncolytic Vaccinia vaccine; NHL, non-Hodgkin lymphoma; PARP, poly(ADP-ribose) polymerase; ns, not significant; BafA1, bafilomycin A1.

terminal disease or excessive tumor growth were euthanized (Fig. 4A). Mice treated with PBS or OVV exhibited larger tumors than mice treated with OVV-Beclin1, R-CHOP or OVV-Beclin1 + R-CHOP, suggesting that OVV-mediated Beclin1 expression significantly suppressed tumor growth and final tumor weight (Fig. 4B-C). Compared with OVV-Beclin1or R-CHOP-alone, combined OVV-Beclin1 + R-CHOP treatment significantly prolonged survival (Fig. 4D).

These results suggested that OVV-Beclin1 treatment was able to confer significant survival benefits. In order to confirm that Beclin1 levels were altered, Beclin1 levels in murine tumor tissue were assessed via IHC. This analysis revealed that tumors from the $\mathrm{PBS}$, OVV and $\mathrm{R}-\mathrm{CHOP}$ groups exhibited less pronounced Beclin1 staining compared with the OVV-Beclin1 and OVV-Beclin1 + R-CHOP groups. This was confirmed this by calculating the frequency of Beclin1-positive cells per unit area relative to that in the PBS control group. H\&E staining was performed to observe the changes of cells in tumor tissue samples: Necrosis was highest in the OVV-Beclin1 + R-CHOP, indicating that the combination of OVV-Beclin1 and OVV-Beclin1 and R-chop exerted a cytotoxic effect (Fig. 4E and F).

\section{Discussion}

A previous study (36) has demonstrated the value of OVV approaches to cancer treatment, as these viruses exhibit superior tumor selectivity and decreased toxicity compared with traditional therapies (37-39). OVV is harmless in normal cells but can effectively kill target tumor cells (40). The OVV genome is large, stable and contains a number of non-essential genes, making it an ideal vector for the delivery of recombinant genes into tumor cells. Tumor-specific OVV strains can be produced by deleting specific virulence genes from the viral genome. The OVV genome is replicated in the cytoplasm of cells, and thus has no significant risk of genomic integration (41). In the present study, the Beclin1 gene was inserted into the OVV genome in a stable manner and these OVV-Beclin1 particles were used to infect NHL cells. Once expressed in tumor cells, Beclin1 induces autophagy and increases the sensitivity of these cells to chemotherapy-induced cell death (42). Beclin1-mediated autophagy can further lead to the autophagic degradation of proteins and organelles essential for chemotherapy resistance, rendering tumor cells more susceptible to DNA damage and death (Fig. 5). 
A

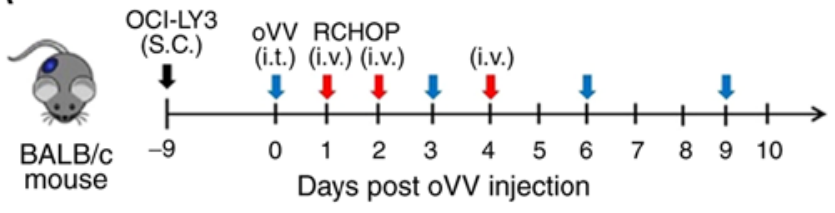

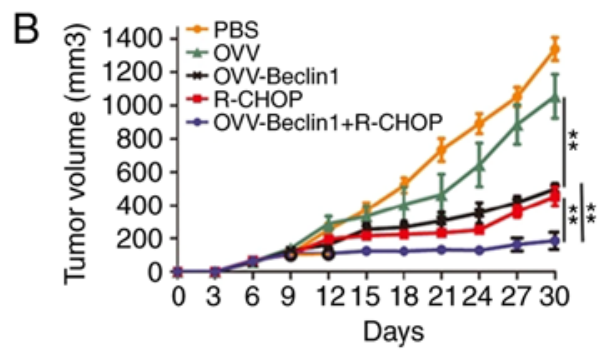

C

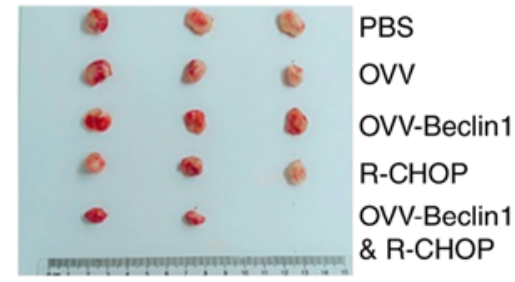

D

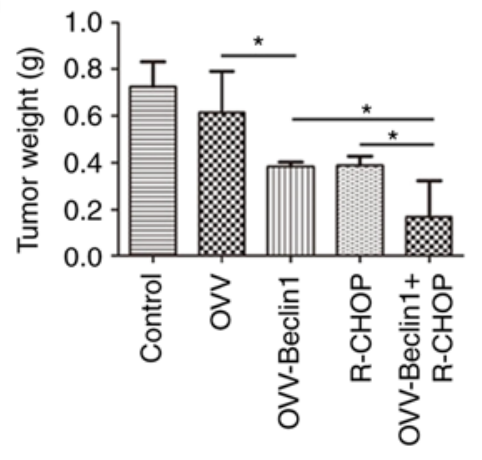

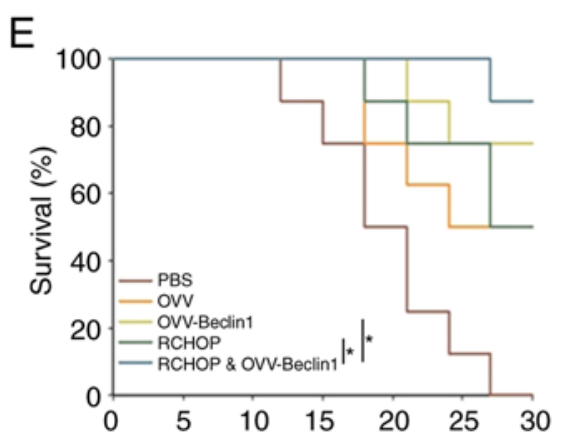

F
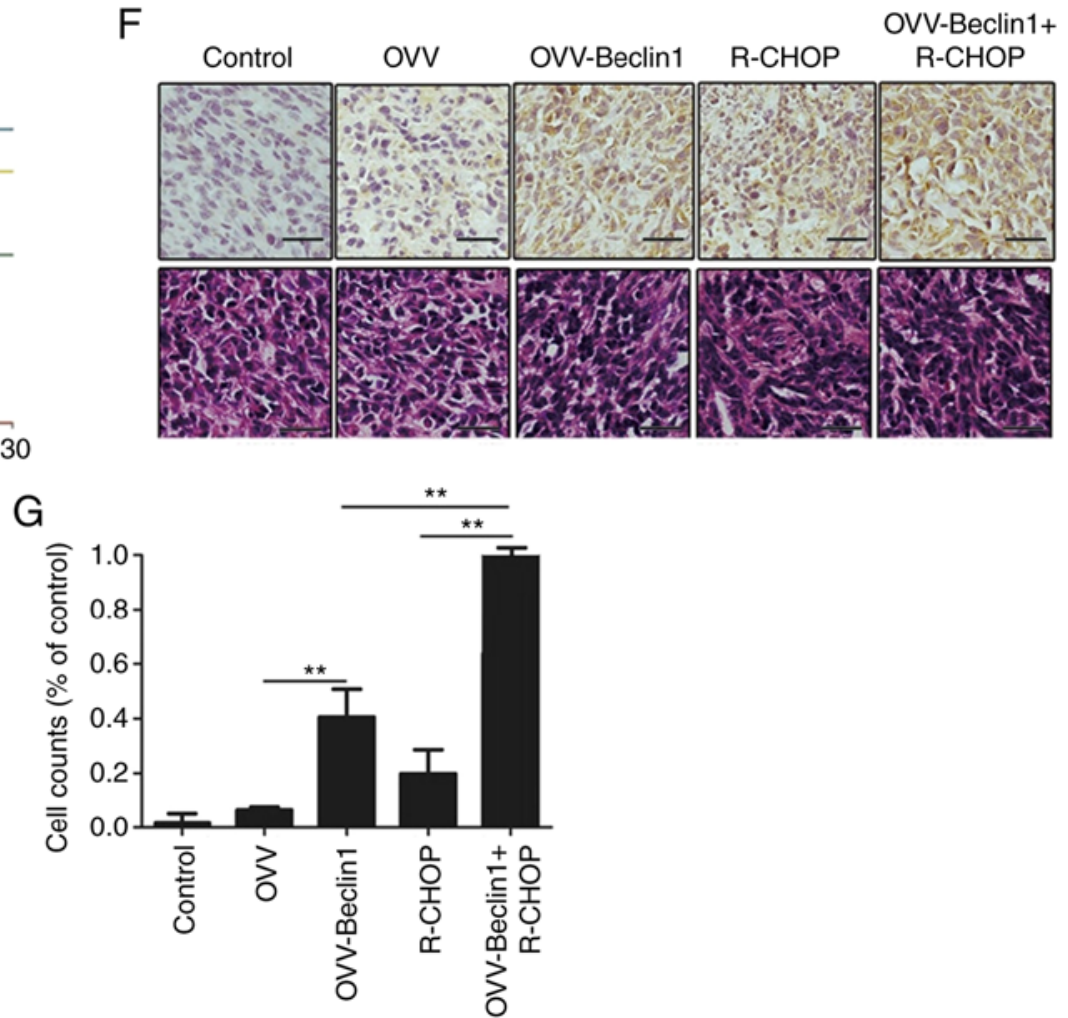

Figure 4. Combination OVV-Beclin1 and R-CHOP inhibits tumor growth in vivo. (A) Establishment of mouse model. A murine non-Hodgkin lymphoma xenograft model was established by implanting BALB/c nude mice with OCI-LY3 cells. Blue arrow, i.t.; red arrow, i.v.; black arrow, S.C. (B) Tumor growth in a murine diffuse large B cell lymphoma xenograft model system was monitored for 30 days. (C) Representative tumor images ( $\mathrm{n}=5$ ). (D) Tumor weight was measured. (E) Murine survival was monitored; mice treated using OVV-Beclin1 exhibited greater survival vs. other treatment groups (P=0.0072). (F) Tumor sections from mice were used for Beclin1 staining; positive cells stained brown. Scale bar, $50 \mu \mathrm{m}$. (G) Beclin1-positive cells relative to PBS control animals were counted. Data are presented as the mean $\pm \mathrm{SD}$. ${ }^{*} \mathrm{P}<0.05$ and ${ }^{* *} \mathrm{P}<0.01$. OVV, oncolytic Vaccinia virus; R-CHOP, Rituximab, Cyclophosphamide, Epirubicin, Vindesine, Prednisolone; i.t., intratumoral; i.v., intravenous; S.C., subcutaneous.

While a previous study focused on the development of therapeutic approaches capable of inducing apoptotic cell death (43), other modes of cell death also serve as an effective anticancer modality. However, induction of autophagic death is not always successful, underscoring the importance of tailoring cancer treatments to specific targets and disease types $(44,45)$. Previous studies have detected Beclin1 downregulation in human esophageal, breast, cervical, lung and liver cancer $(46,47)$. The upregulation of Beclin1 can induce excessive autophagy in leukemia cells, leading to their death (6). Here, the functional role of Beclin1 in NHL was investigated; Beclin1 was downregulated in this cancer type suggesting that these cells may be sensitive to autophagic induction. These findings are consistent with work by Huang et al (48) demonstrating that 


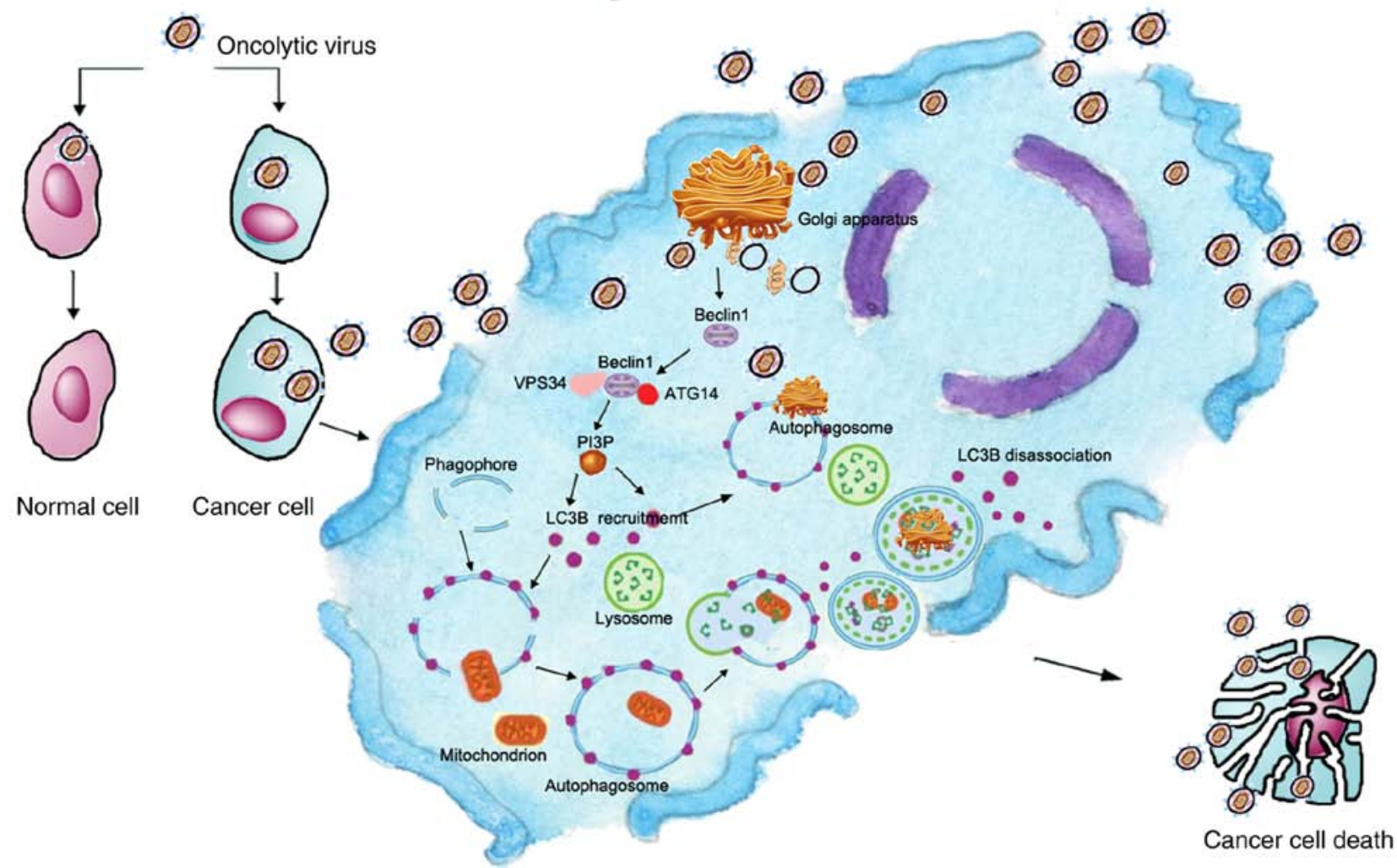

Figure 5. Mechanism of oncolytic viruses. OVV identifies and enters tumor cells. OVV replication in the cytoplasm induces autophagosome formation. These bilayered structures surround chemotherapy-damaged proteins and organelles, preventing normal functioning of the tumor cell. Finally, the tumor cell bursts and OVV is released. OVV, oncolytic Vaccinia virus; ATG14, autophagy-related 14.

$85 \%$ of patients with DLBCL exhibited decreased Beclin1 protein levels following R-CHOP treatment.

The present study developed an OVV-Beclin1 vector which could be used to infect NHL tumor cells and induce excessive autophagy, culminating in cell death. Our previous research showed that OVV-Bclin1 exhibits good therapeutic effects on leukemia and myeloma via dissolving tumor cells and inducing autophagy death (30). Additionally, previous work has shown that Beclin1 upregulation in DLBCL increases PI3KC3 expression and associated phosphorylated-AKT1 activation. Beclin1 interacts with PI3KC3 and then with autophagy-related 12,5 and $16 \mathrm{~L}$ and $\mathrm{LC} 3 \mathrm{~B}$ to facilitate the gradual expansion and maturation of autophagosomes within the cell (49). Autophagy has been found to serve a role in $\mathrm{R}$-CHOP resistance in patients with lymphoma, suggesting that inducing autophagy may be a valuable therapeutic strategy (30).

The present study was able to confirm that OVV-Beclin1 induced autophagic death of NHL cells, indicated by detection of autophagosome formation in these cells. OVV-Beclin1-treated cells did not undergo apoptotic death and dose-dependent increases in virus-induced cytotoxicity were not associated with increases in apoptotic staining or protein levels. At low levels, the induction of autophagy can help tumor cells resist treatment by degrading damaged organelle and proteins. However, excessive autophagy can lead to unrestrained destruction of functional proteins and organelles, compromising cell viability and leading to tumor cell death (50). Here, OVV-Beclin1-induced autophagic death also resulted in the release of viral particles that can then infect proximal tumor cells, leading to further cell death and enhanced R-CHOP efficacy.

\section{Acknowledgements}

Not applicable.

\section{Funding}

The present study was supported by the Zhejiang Medical Technology Plan Project (grant nos. WKJ-ZJ-1709 and 2020KY052), National Science Foundation of China (grant nos. 81570198 and 81602706) and Zhejiang Provincial Natural Science Foundation of China (grant nos.LY19H160037 and LY17H160062).

\section{Availability of data and materials}

All data generated or analyzed in this study are included in this published article.

\section{Authors' contributions}

SW, YW, WL and XT designed the study. SX, WF, CY, HP and YW performed the experiments and collected and analyzed data. SX, WF and HP wrote, reviewed and revised the manuscript. All authors read and approved the final version of the manuscript. 


\section{Ethics approval and consent to participate}

All animal experiments were performed in accordance with ethical standards of the Institutional Animal Use and Care Committee of the Zhejiang Provincial People's Hospital, and ethical approval (approval no. A20190029) was obtained prior to the commencement of the study.

\section{Patient consent for publication}

Not applicable.

\section{Competing interests}

The authors declare that they have no competing interests.

\section{References}

1. Howlader N, Morton L, Feuer E, Besson C and Engels E: Contributions of subtypes of non-hodgkin lymphoma to mortality trends. Cancer Epidemiol Biomarkers Prev 25: 174-179, 2016.

2. Coccaro N, Anelli L, Zagaria A, Perrone T, Specchia G and Albano F: Molecular complexity of diffuse large b-cell lymphoma: Can it be a roadmap for precision medicine? Cancers 12: 185, 2020.

3. Camicia R, Winkler HC and Hassa PO: Novel drug targets for personalized precision medicine in relapsed/refractory diffuse large B-cell lymphoma: A comprehensive review. Mol Cancer 14: $207,2015$.

4. Xu-Monette ZY, Wu L, Visco C, Tai YC, Tzankov A, Liu Wm, Montes-Moreno S, Dybkaer K, Chiu A, Orazi A, et al: Mutational profile and prognostic significance of TP53 in diffuse large B-cell lymphoma patients treated with R-CHOP: Report from an international DLBCL rituximab-CHOP consortium program study. Blood 120: 3986-3996, 2012.

5. Lee D, Lee D, Hwang Y, Seo H, Lee S and Kwon J: The bromodomain inhibitor PFI-3 sensitizes cancer cells to DNA damage by targeting SWI/SNF. Mol Cancer Res 18: 0289, 2020

6. Wechman SL, Pradhan AK, DeSalle R, Das SK, Emdad L, Sarkar D and Fisher PB: New insights into beclin-1: Evolution and pan-malignancy inhibitor activity. Adv Cancer Res 137: 77-114, 2018.

7. Roy DG and Bell JC: Cell carriers for oncolytic viruses: Current challenges and future directions. Oncolytic Virother 2: 47-56, 2013.

8. Aitken AS, Roy DG and Bourgeois-Daigneault MC: Taking a stab at cancer; oncolytic virus-mediated anti-cancer vaccination strategies. Biomedicines 5: 3, 2017.

9. Willmon C, Harrington K, Kottke T, Prestwich R, Melcher A and Vile R: Cell carriers for oncolytic viruses: Fed ex for cancer therapy. Mol Ther 17: 1667-1676, 2009.

10. Amin AD, Peters TL, Li L, Rajan SS, Choudhari R, Puvvada SD and Schatz JH: Diffuse large B-cell lymphoma: Can genomics improve treatment options for a curable cancer? Cold Spring Harb Mol Case Stud 3: a001719, 2017.

11. Huang F, Wang BR and Wang YG: Role of autophagy in tumorigenesis, metastasis, targeted therapy and drug resistance of hepatocellular carcinoma. World J Gastroenterol 24: 4643-4651, 2018.

12. Denton D, Nicolson S and Kumar S: Cell death by autophagy: Facts and apparent artefacts. Cell Death Differ 19: 87-95, 2012.

13. Puri $\mathrm{P}$ and Chandra A: Autophagy modulation as a potential therapeutic target for liver diseases. J Clin Exp Hepatol 4: 51-59, 2014.

14. Galluzzi L, Pietrocola F, Bravo-San Pedro JM, Amaravadi RK, Baehrecke EH, Cecconi F, Codogno P, Debnath J, Gewirtz DA Karantza V, et al: Autophagy in malignant transformation and cancer progression. EMBO J 34: 856-880, 2015.

15. Correa RJM, Valdes YR, Shepherd TG and DiMattia GE: Beclin-1 expression is retained in high-grade serous ovarian cancer yet is not essential for autophagy induction in vitro. J Ovarian Res 8: $1-15,2015$.

16. Hasui K, Wang J, Jia X, Tanaka M, Nagai T, Matsuyama T and Eizuru Y: Enhanced autophagy and reduced expression of cathepsin d are related to autophagic cell death in epstein-barr virus-associated nasal natural killer/T-cell lymphomas: An immunohistochemical analysis of beclin-1, LC3, mitochondria (AE-1), and cathepsin D in nasopharyngeal lymphomas. Acta Histochem Cytochem 44: 119-131, 2011.
17. Guaman-Ortiz LM, Orellana MIR and Ratovitski EA: Natural compounds as modulators of non-apoptotic cell death in cancer cells. Curr Genom 18: 132-155, 2017.

18. Shen HM and Codogno P: Autophagic cell death: Loch ness monster or endangered species? Autophagy 7: 457-465, 2011.

19. Sui X, Chen R, Wang Z, Huang Z, Kong N, Zhang M, Han W, Lou F, Yang J, Zhang Q, et al: Autophagy and chemotherapy resistance: A promising therapeutic target for cancer treatment. Cell Death Dis 4: e838, 2013.

20. Derenzini E, Agostinelli C, Imbrogno E, Iacobucci I, Casadei B Brighenti E, Righi S, Fuligni F, Di Rorà AGL, Ferrari A, et al: Constitutive activation of the DNA damage response pathway as a novel therapeutic target in diffuse large B-cell lymphoma. Oncotarget 6: 6553-6569, 2015.

21. Czarny P, Pawlowska E, Bialkowska-Warzecha J, Kaarniranta K and Blasiak J: Autophagy in DNA damage response. Int J Mol Sci 16: 2641-26621, 2014.

22. Zhang D, Tang B, Xie X, Xiao YF, Yang SM and Zhang JW: The interplay between DNA repair and autophagy in cancer therapy. Cancer Biol Ther 16: 1005-1013, 2014.

23. Mizushima N. Autophagy: Process and function. Genes Dev 21: 2861-2873, 2007.

24. Rojas JJ and Thorne SH: Theranostic potential of oncolytic vaccinia virus. Theranostic 2: 363-373, 2012.

25. Quirin C, Rohmer S, Fernández-Ulibarri I, Behr M, Hesse A, Engelhardt S, Erbs P, Enk AH and Nettelbeck DM: Selectivity and efficiency of late transgene expression by transcriptionally targeted oncolytic adenoviruses are dependent on the transgene insertion strategy. Hum Gene Ther 22: 389-404, 2007.

26. Bastin D, Walsh SR, Saigh MA and Wan Y: Capitalizing on cancer specific replication: Oncolytic viruses as a versatile platform for the enhancement of cancer immunotherapy strategies. Biomedicines 4: 21, 2016.

27. Haddad D: Genetically engineered vaccinia viruses as agents for cancer treatment, imaging, and transgene delivery. Front Oncol 7: 96, 2017.

28. Buijs PR, Verhagen JH, van Eijck CH and van den Hoogen BG: Oncolytic viruses: From bench to bedside with a focus on safety. Hum Vaccin Immunother 11: 1573-1584, 2007.

29. Deng L, Fan J, Ding Y, Zhang J and Huang B: Oncolytic efficacy of thymidine kinase-deleted vaccinia virus strain guang9. Oncotarget 8: 40533-40543, 2017.

30. Lei W, Wang S, Xu N, Chen Y, Wu G, Zhang A, Chen X, Tong Y and Qian W: Enhancing therapeutic efficacy of oncolytic vaccinia virus armed with beclin-1, an autophagic gene in leukemia and myeloma. Biomed Pharmacother 125: 110030, 2020.

31. Tong Y, You L, Liu H, Li L, Meng H, Qian Q and Qian W: Potent antitumor activity of oncolytic adenovirus expressing beclin-1 via induction of autophagic cell death in leukemia. Oncotarget 4: 860-874, 2013.

32. Yu HC, Hou DR, Liu CY, Lin CS, Shiau CW, Cheng AL and Chen KF: Cancerous inhibitor of protein phosphatase 2A mediates bortezomib-induced autophagy in hepatocellular carcinoma independent of proteasome. PLoS One 8: e55705, 2013.

33. Diaz SL: Conducting and reporting animal experimentation: Quo vadis? Eur J Neurosci 52: 3493-3498, 2020.

34. Nguyen T, Shaid S, Vakhrusheva O, Koschade SE, Klann K, Thölken M, Baker F, Zhang J, Oellerich T, Sürün D, et al: Loss of the selective autophagy receptor p62 impairs murine myeloid leukemia progression and mitophagy. Blood 133: 168-179, 2019.

35. Araveti PB and Srivastava A: Curcumin induced oxidative stress causes autophagy and apoptosis in bovine leucocytes transformed by. Cell Death Discov 5: 100, 2019.

36. Islam SM, Lee B, Jiang F, Kim E, Ahn S and Hwang TH: Engineering and characterization of oncolytic vaccinia virus expressing truncated herpes simplex virus thymidine kinase. Cancers 12: 228, 2020.

37. Walsh S, Bastin D, Chen L, Nguyen A, Storbeck CJ, Lefebvre C, Stojdl D, Bramson JL, Bell JC and Wan Y: Type I IFN blockade uncouples immunotherapy-induced antitumor immunity and autoimmune toxicity. J Clin Invest 129: 518-530, 2019.

38. Zhang B and Cheng P: Improving antitumor efficacy via combinatorial regimens of oncolytic virotherapy. Mol Cancer 19: 158, 2020.

39. Bourgeois-Daigneault M, Roy DG, Aitken AS, El Sayes E, Martin NT, Varette O, Falls T, St-Germain LE, Pelin A, Lichty $\mathrm{BD}$, et al: Neoadjuvant oncolytic virotherapy before surgery sensitizes triple-negative breast cancer to immune checkpoint therapy. Sci Transl Med 10: 422, 2018. 
40. Guo ZS, Lu B, Guo Z, Giehl E, Feist M, Dai E, Liu W, Storkus WJ, He Y, Liu Z and Bartlett DL: Vaccinia virus-mediated cancer immunotherapy: Cancer vaccines and oncolytics. J Immunother Cancer 7: 6, 2019.

41. Liu Z, Ge Y, Wang H, Ma C, Feist M, Ju S, Guo ZS and Bartlett DL: Modifying the cancer-immune set point using vaccinia virus expressing re-designed interleukin-2. Nat Commun 9: 4682 , 2018.

42. Hill SM, Wrobel L and Rubinsztein DC: Post-translational modifications of Beclin 1 provide multiple strategies for autophagy regulation. Cell Death Differ 26: 617-629, 2019.

43. Place DE and Kanneganti TD: Cell death-mediated cytokine release and its therapeutic implications. J Exp Med 216 1474-1486, 2019.

44. Torii S, Honda S, Murohashi M, Yamaguchi H and Shimizu S Autophagy involvement in oncogenesis. Cancer Sci 111: 3993-3999, 2020.

45. Levy JM and Thorburn A: Autophagy in cancer: Moving from understanding mechanism to improving therapy responses in patients. Cell Death Differ 27: 843-857, 2020.

46. Liu G, Pei F, Yang F, Li L, Amin AD, Liu S, Buchan JR and Cho WC: Role of autophagy and apoptosis in non-small-cell lung cancer. Int J Mol Sci 18: 367, 2017.
47. Zhang J, Yang S, Wang K, Huang Y, Yang N, Yang Z, Zheng Z and Wang Y: Crocin induces autophagic cell death and inhibits cell invasion of cervical cancer $\mathrm{SiHa}$ cells through activation of PI3K/AKT. Ann Transl Med 8: 1180, 2020.

48. Huang JJ, Zhu YJ, Lin TY, Jiang WQ, Huang HQ and Li ZM: Beclin 1 expression predicts favorable clinical outcome in patients with diffuse large B-cell lymphoma treated with R-CHOP. Hum Pathol 42: 1459-1466, 2011

49. Wang Z, Zhu S, Zhang G and Liu S: Inhibition of autophagy enhances the anticancer activity of bortezomib in B-cell acute lymphoblastic leukemia cells. Am J Cancer Res 5: 639-650, 2015.

50. Zhu Y, Jia H, Gao G, Pan GY, Jiang YW, Li P, Zhou N, Li C, She C, Ulrich NW, et al: Mitochondria-acting nanomicelles fordestruction of cancer cells via excessive mitophagy/autophagy-driven lethal energy depletion and phototherapy. Biomaterials 232: 119668 , 2020 .

This work is licensed under a Creative Commons Attribution-NonCommercial-NoDerivatives 4.0 International (CC BY-NC-ND 4.0) License. 